\title{
Distribution and phenotype of Epstein-Barr virus-infected cells in human pharyngeal tonsils
}

\author{
S David Hudnall ${ }^{1,2}$, Yimin $\mathrm{Ge}^{1}$, Longxing Wei ${ }^{1,2}$, Ning-Ping Yang ${ }^{3}$, Hui-Quin Wang ${ }^{3}$ \\ and Tiansheng Chen ${ }^{1,2}$
}

${ }^{1}$ Department of Pathology and Laboratory Medicine, University of Texas Medical Branch, Galveston, TX, USA $;^{2}$ The WHO Collaborating Center for Tropical Diseases, University of Texas Medical Branch, Galveston, TX, USA and ${ }^{3}$ Sealy Center for Environmental Health and Medicine, University of Texas Medical Branch, Galveston, TX, USA

\begin{abstract}
Although Epstein-Barr virus (EBV) is often found in human tonsils, it remains to be precisely determined in what cells and microenvironment the virus is present. Although generally regarded as a $B$ lymphotropic virus, EBV is associated with non-B-cell tumors, for example, NK/T-cell lymphoma, carcinoma, and leiomyosarcoma. To provide a basis for understanding the origin and biology of EBV-infected non-B cells, the immunophenotype of all EBV-infected cells in reactive human tonsils was determined by subjecting tonsil sections to dual/triple EBER in situ hybridization and immunohistochemistry with monoclonal antibodies to T cells (CD3, CD4, CD8, CCR3), B cells (CD20), plasma cells (CD138), natural killer (NK) cells (PEN5), and epithelial cells (cytokeratin), as well as frozen section immunostaining with antibodies to EBV latent proteins EBNA1, EBNA2, LMP1, and EBV early protein BZLF1. Most tonsils contained nearly equal numbers of EBNA1- and LMP1-positive cells (latency program) while only a few contained EBNA2-positive cells (growth program). More than 1000 EBER-positive cells from six tonsils were detected in the interfollicular zone (59\%), tonsillar crypts (26\%), and follicles (15\%). Most (82\%) EBER-positive cells are CD20-positive B cells, $7 \%$ are CD3-positive T cells, and $11 \%$ are cells of indeterminate lineage, often with plasmacytoid morphology. However, no EBER-positive plasma cells were identified. Rare EBER-positive NK cells and EBER/BZLF1-positive epithelial cells were identified. The direct demonstration of EBV within rare T cells, NK cells, and epithelial cells in reactive human tonsils provide a basis for further understanding of the origin of EBV-associated tumors of non-B-cell type.

Modern Pathology (2005) 18, 519-527, advance online publication, 4 February 2005; doi:10.1038/modpathol.3800369
\end{abstract}

Keywords: Epstein-Barr virus; tonsil; lymphoid tissue; lymphocytes; immunohistochemistry; in situ hybridization

Based upon serologic surveys, more than $90 \%$ of adults worldwide have been infected by the Epstein-Barr virus (EBV). ${ }^{1}$ Nearly all infection is acquired by oral contact with a person carrying EBV in saliva. ${ }^{2}$ Although the exact details of virus transmission in the oral cavity remain unknown, it is likely that initial infection of either oral epithelial cells or tonsillar B cells is followed by a brief period of replication and lifelong persistence in B lymphocytes. ${ }^{3}$ In one study, no EBV-infected epithelial cells were identified in tonsils removed during infectious mononucleosis, strongly suggesting that EBV infec-

Correspondence: Dr SD Hudnall, MD, Department of Pathology, University of Texas Medical Branch, 301 University Blvd., Galveston, TX 77555-0741, USA.

E-mail: shudnall@utmb.edu

Received 28 July 2004; revised 24 October 2004; accepted 10

November 2004; published online 4 February 2005 tion of epithelial cells, if it occurs at all, must be a rare event. ${ }^{4}$ It has been suggested that B cells might encounter salivary EBV directly through the crypt epithelium, thus bypassing the need for virus transmission from epithelial cell to B lymphocyte. ${ }^{5}$ It has been suggested that newly infected tonsil B cells first migrate to germinal centers where they proliferate under the influence of the full complement of latent viral proteins, ${ }^{6}$ followed by persistence as long-lived memory $B$ cells with viral gene expression limited to LMP2a. ${ }^{7}$

In the present study, formalin-fixed paraffinembedded sections of EBV-positive human tonsils surgically removed for tonsillar enlargement were subjected to combined immunohistochemistry and EBER in situ hybridization (ISH) to localize EBV within various phenotypically defined cell types, including CD3-positive $\mathrm{T}$ cells, CD20-positive B cells, PEN5-positive natural killer (NK) cells, 
CD138-positive plasma cells, and cytokeratin-positive epithelial cells. To characterize EBV gene expression in infected cells, immunohistochemical staining for EBV latent proteins EBNA1, EBNA2, and LMP2a on frozen sections, and the immediateearly protein BZLF1 on paraffin sections was also performed.

\section{Materials and methods}

\section{Tonsil Specimens}

Fresh frozen tissue from approximately 50 tonsils was first screened by EBNA1 PCR, of which $>90 \%$ were positive. A randomly chosen subset of 20 was then selected for EBER ISH (on paraffin sections). The age range of the tonsil donors was 5-57 years (mean age 24 years), with a male/female ratio of 1.2 and a $50 \%$ white, $30 \%$ black, and $20 \%$ Hispanic ethnic distribution. While most tonsils tested contained fewer than 10 EBER + cells per tissue section, after much searching we identified six with 50-150 positive cells per section. These tonsils were selected for dual or triple EBER ISH and immunohistochemistry with a fluorescein-labeled EBER1 oligonucleotide probe, and monoclonal antibodies to T cells (CD3, CD4, CD8, CCR3), B cells (CD20), plasma cells (CD138), NK cells (PEN5), and epithelial cells (pan-cytokeratin). EBV protein expression was detected by frozen section immunohistochemistry with antibodies to latent proteins EBNA1, EBNA2, LMP1, and LMP2a, and by paraffin section immunohistochemistry with antibody to the immediate-early protein BZLF1 (ZEBRA).

\section{EBNA1 PCR}

DNA was extracted from fresh frozen tonsil tissue (QIAamp DNA Midi kit, Qiagen, Valencia, CA, USA) and subjected to nested PCR using two sets of nonoverlapping primers to the EBNA1 gene (external: 5'-CCTGTAGGGGAAGCCGAT and 5'CAATGGTGTAAGACGACATT, internal: 5'-CCAA GAAGGTGGCCCAGA and 5'-CCTGCCTCCATCAC CCTG). A $387 \mathrm{bp}$ external PCR product and a $120 \mathrm{bp}$ internal PCR product were obtained. Nested PCR products were detected by chemiluminescent dot blot hybridization with a digoxigenin $3^{\prime}$-endlabeled (Roche Diagnostics, Indianapolis, IN, USA) oligonucleotide probe (5'-ACGCATGCATCTCCCTGT).

\section{EBER ISH}

Deparaffinized sections of EBV-infected tonsillar tissue were pretreated with proteinase $\mathrm{K}$ for $20 \mathrm{~min}$ and incubated with fluorescein-conjugated EBER PNA probe (Dako, Carpinteria, CA, USA) at $55^{\circ} \mathrm{C}$ for $2-4 \mathrm{~h}$. The sections were rinsed in water and incubated with alkaline phosphatase-conjugated anti-fluorescein antibody for $30 \mathrm{~min}$ before adding fresh NBT color substrate (bromochloroindole phosphate and nitroblue tetrazolium) to produce an alcohol-insoluble dark blue intranuclear stain in EBV-positive cells.

\section{Immunohistochemistry}

Immediately following EBER ISH, the sections were subjected to antigen retrieval in citrate buffer $\mathrm{pH} 6$ at $95^{\circ} \mathrm{C}$ for $20 \mathrm{~min}$. The sections were allowed to cool to room temperature for $20 \mathrm{~min}$, and rinsed in water. After blocking with avidin and biotin, the sections were incubated for $1 \mathrm{~h}$ with one of the following unconjugated monoclonal antibodies to human antigens CD3, CD20, CD138 (Dako, Carpinteria, CA, USA), CD4, CD8 (Serotec, Raleigh, NC, USA), CCR3 (BD Biosciences Pharmingen, San Diego, CA, USA), PEN5 (5H10 clone, gift of E Vivier, Centre d'Immunologie, INSERM-CNRS, Marseille, France), or cytokeratin (AE1/AE3, Dako); and EBV antigens EBNA2 (PE2, Dako), BZLF1 (Dako), LMP1 (Dako), and EBNA1 (gift of $\mathrm{E}$ Kremmer, GSF-Institut für Molekulare Immunologie, Munich, Germany). DAKO link or USA link (Signet, Dedham, MA, USA) were used for secondary signal amplification, followed by either diaminobenzidine (brown) or fast red (pink) color development. For triple labeling following EBER ISH, the first primary antibody was developed with diaminobenzidine followed by second primary antibody development with fast red (Envision Double Stain Kit, Dako).

\section{Light Microscopy}

The localization and lineage of all EBER-positive cells (>1000 cells) from paraffin-embedded formalin-fixed tonsil sections was determined by colorimetric EBER ISH followed by single or double immunohistochemical staining with well-characterized antibodies to cell lineage markers CD3 ( $\mathrm{T}$ cells), CD4 ( $\mathrm{T}$ cell subset), CD8 ( $\mathrm{T}$ cell subset), CD20 (B cells), CD138 (plasma cells), PEN5 (NK cells), and cytokeratin (epithelial cells). EBV protein expression was examined by immunohistochemical staining of frozen sections for EBV latent antigens EBNA-1, EBNA-2, LMP-1, LMP-2a, and the immediate-early antigen BZLF-1.

\section{Results}

\section{Distribution and Morphology of EBV-Infected Tonsil Cells}

Nearly $60 \%$ of the EBER-positive cells were found within the T-cell-rich interfollicular regions, while $27 \%$ were present within the crypt epithelium, and $15 \%$ were present within the B-cell-rich germinal centers of secondary follicles (Table 1, Figure 1a-c). In contrast to the relatively large number of EBERpositive cells in the crypt epithelium, none were 
Table 1 Location and immunophenotype of 1191 EBER-positive human tonsil cells

\begin{tabular}{|c|c|c|c|c|}
\hline & Intraepithelial (\%) & Interfollicular (\%) & Follicular (\%) & Total (\%) \\
\hline CD20+ B cells & 24 & 44.2 & 14 & 82.2 \\
\hline CD3+ T cells & 0.3 & 6.4 & 0 & 6.7 \\
\hline Cytokeratin+epithelial cells & 1.2 & - & - & 1.2 \\
\hline Null cells ${ }^{\mathrm{a}}$ & 1.3 & 8.4 & 1.2 & 10.9 \\
\hline Total & 26.8 & 59 & 15.2 & 100 \\
\hline
\end{tabular}

${ }^{\mathrm{a}}$ Many of the null cells express extremely dim CD20 and have plasmacytoid features, and thus are likely to be plasmacytoid B cells.

identified within the stratified squamous epithelium of the tonsillar surface. Individual EBERpositive cells appeared to be randomly distributed within the interfollicular and crypt epithelial regions, whereas in germinal centers the distribution appeared to be nonrandom, with a few germinal centers containing several positive cells. No EBERpositive cells were seen within follicular mantle zone regions or salivary glandular elements. Most (>90\%) EBER-positive cells had morphologic features consistent with small mature lymphocytes (Figure 1c,d), some with plasmacytoid features, while a few had features of large transformed lymphoid cells.

\section{Immunophenotype of EBV-Infected Tonsil Cells}

Most (82\%) of the EBER-positive tonsil cells very weakly expressed the B-cell marker CD20, while $11 \%$ did not clearly express any of the cell lineage markers for B cells, T cells, plasma cells, NK cells, or epithelial cells (Table 1). In contrast to CD20, only $11 \%$ of EBER-positive cells stained with antibody to the B-cell-specific antigen CD79a in the single case tested. Since many of the small cells of indeterminate phenotype had plasmacytoid features, and at least some appeared to express very dim CD20, many of these cells may be plasmacytoid B cells. However, staining with the plasma cell marker CD138 failed to identify any dual EBER/CD138positive plasma cells (Figure 1n). While EBERpositive B cells were often found tightly bound to T cells (Figure 1d,e), only a small number $(7 \%)$ of dual EBER/CD3-positive $\mathrm{T}$ cells were identified (Figure 1f). Many of these EBER-positive T cells appear to be CD4 positive, while only rare CD8positive cells were identified (Table 2, Figure 1g, h). Despite the presence of numerous PEN5-positive NK cells just beneath the crypt epithelium (Figure 1i), dual EBER-PEN5-positive NK cells were identified only very rarely, and appeared small, contracted and apoptotic. Initial attempts to utilize the alternative NK cell marker CD56 with EBER in situ were unsuccessful. It is likely that the protease digestion required for EBER in situ along with the normally weak reactivity of anti-CD56 in paraffin sections lead to poor staining. Although most of the EBERpositive cells within the crypt epithelium appeared as small lymphocytes (Figure 1j), rare dual EBERcytokeratin-positive crypt epithelial cells were found (Figure 1k).

\section{EBV Gene Expression}

Matched frozen sections of 20 individual tonsils were immunostained for EBV latent antigens EBNA1 (Figure 1o), EBNA2 (Figure 1p), and LMP1 (Figure 1q). The number of positive cells expressing each latent antigen in matching sections was recorded to compare expression patterns of viral latent proteins in these tissues (Table 3). Scattered EBNA1-positive cells were detected in 19 of 20 tonsils, with counts ranging from 2 to 90 cells per section. EBNA1 and LMP1 were coexpressed in all 15 tonsils examined. In six of 15 tonsils, significantly more LMP1-positive cells than EBNA1positive cells were detected. In contrast to the near-universal expression of EBNA1 and LMP1, EBNA2-positive cells were detected in only nine of 19 EBNA1-positive tonsils. However, when present, the number of EBNA2-positive cells equaled or exceeded the number of EBNA1-positive cells in nearly every case. A striking finding in these tonsils was the 'on-off' character to EBNA2 expression, i.e. either no EBNA2-positive cells or many EBNA2positive cells were present in each tonsil. Most of the EBV-infected cells in these non-IM tonsils thus appear to express the highly restricted EBNA2negative form of latency termed the 'latency program' characteristic of either differentiating germinal center B cells or differentiated memory B cells, rather than the EBNA2-positive 'growth program' characteristic of recently infected lymphoblastoid cells produced by in vitro infection of peripheral blood B cells. ${ }^{6}$ Intranuclear BZLF1positivity, indicative of EBV lytic activation, was detected in all tonsils examined. Nearly all of the BZLF1-positive cells were small lymphocytes just below or within the tonsillar crypt epithelium, many with plasmacytoid morphologic features (Figure 1l). In some cases, rare BZLF1-positive epithelial cells were also detected (Figure 1m). 

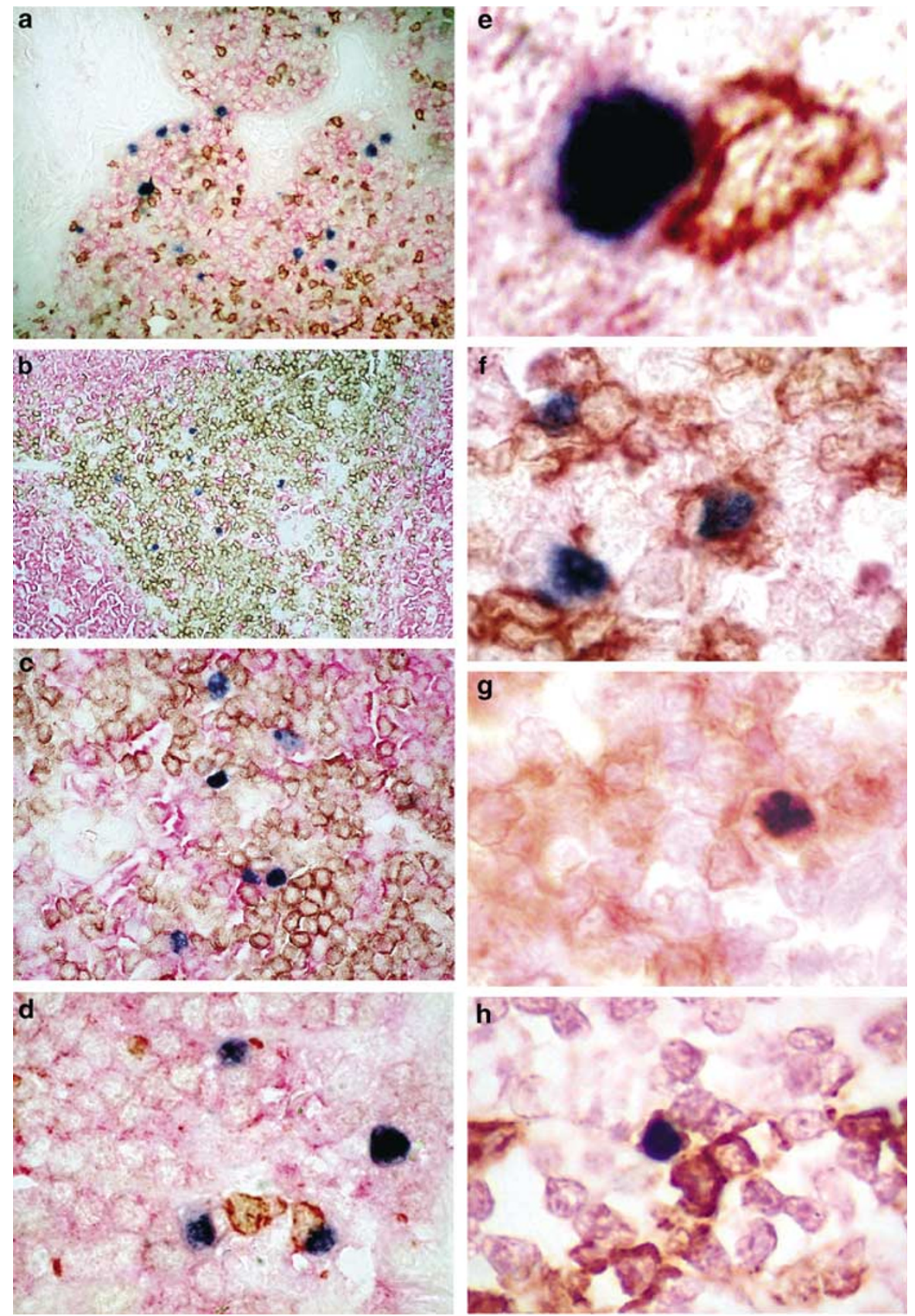

Figure 1 (a) Numerous EBER-positive (blue) CD20-positive (pink) B cells just beneath the unstained tonsil crypt epithelium (EBER ISH with dual CD3/CD20 immunostain). Most of the lymphocytes are CD20-positive B cells with scattered CD3-positive T cells. (b) Numerous scattered EBER-positive B cells within the T-cell-rich interfollicular zone. Note the B-cell-rich follicles in the periphery (triple EBER ISH/ CD3/CD20 stain). (c) High-power view of EBER-positive B cells in a T-cell-rich region (triple EBER ISH/CD3/CD20 stain). (d) Conjugate formation of CD3-positive T cells with EBER-positive B cells (triple EBER ISH/CD3/CD20 stain). (e) High-power view of tight binding between an EBER-positive B cell (left) and a CD3-positive T cell (right) (triple EBER ISH/CD3/CD20 stain). (f) EBER-positive T cell (center) (triple EBER ISH/CD3/CD20 stain). (g) EBER-positive CD4-positive T cell (dual EBER ISH/CD4 stain). (h) EBER-positive CD8-positive T cell (dual EBER ISH/CD8 stain). (i) NK cells (brown) and EBER-positive cells (blue) just beneath the crypt surface (upper left) (dual EBER ISH/PEN5 stain). (j) Small EBER-positive lymphoid cells in close association with the reticulated crypt epithelium (brown). Note the numerous lymphoid cells admixed within the crypt epithelium (dual EBER ISH/cytokeratin stain). (k) Two EBER-positive crypt epithelial cells (dual EBER ISH/cytokeratin stain). (l) Scattered BZLF1-positive cells (brown) with plasmacytoid features within the lymphocyte-rich crypt epithelium (BZLF1 immunostain). (m) Two BZLF1-positive epithelial cells (brown) in tonsillar crypt. Note the flattened appearance typical of a surface epithelial cell displayed by the positive cell in the upper field. Closer examination of the round cell in lower field reveals numerous intercellular desmosomes indicative of epithelial cell origin (BZLF1 immunostain). (n) Numerous large CD138-positive plasma cells (brown) and a single small EBER-positive lymphoid cell located just beneath the crypt epithelium (dual EBER ISH/CD138 stain). (o) Cluster of (brown) EBNA1-positive lymphoid cells (EBNA1 immunostain on frozen section). (p) Cluster of (brown) EBNA2-positive lymphoid cells (EBNA2 immunostain on frozen section). (q) Cluster of (brown) LMP1-positive lymphoid cells. Note that in this field some of the cell debris seems to have been engulfed by resident macrophages (LMP1-4 immunostain). (r) Single BZLF1-positive spindle cell (brown) in the tonsillar stroma (BZLF1 immunostain). 

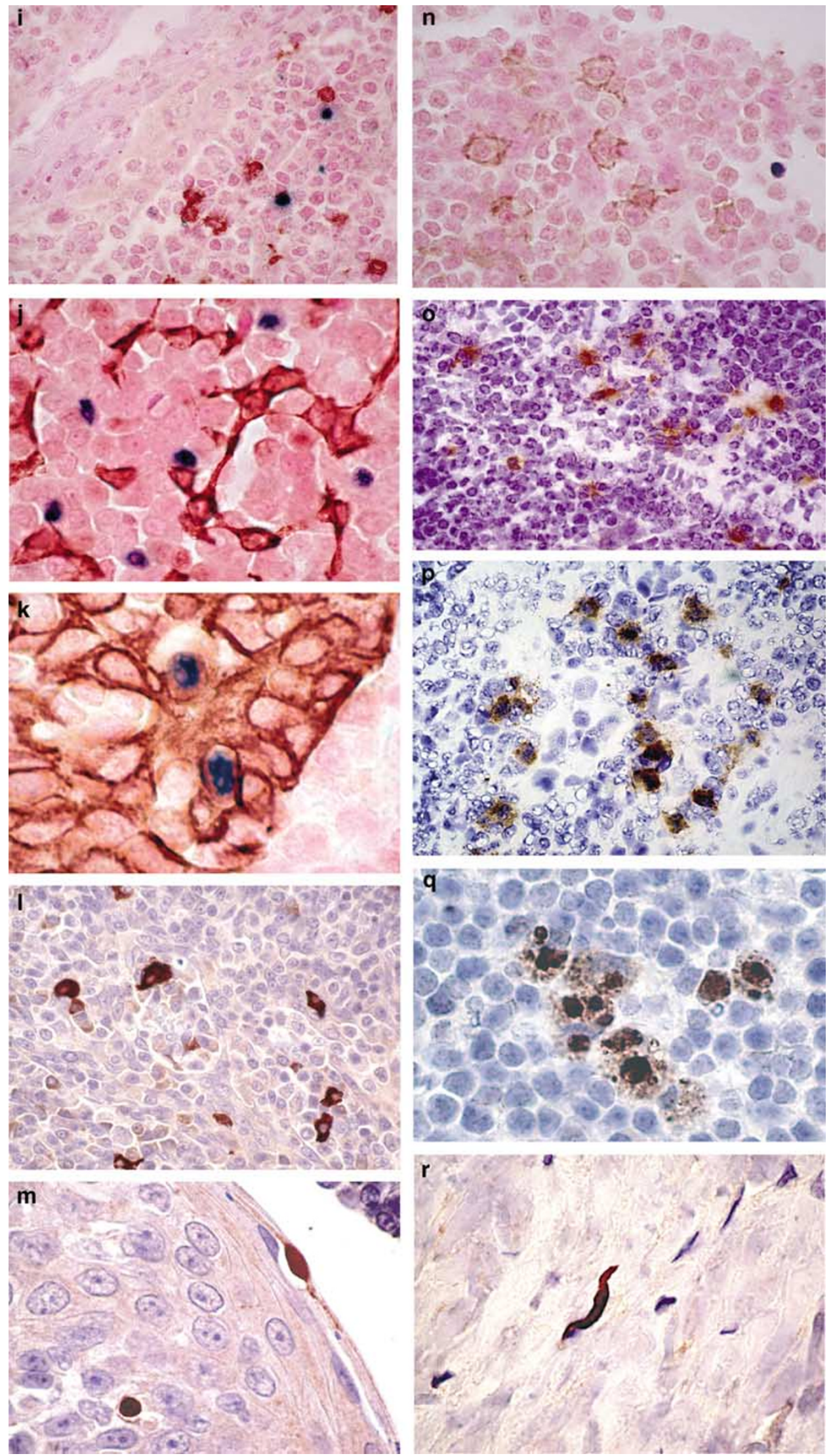

Figure 1 Continued. 


\section{EBER + B-Cell-T-Cell Conjugates}

Sections of EBV-positive tonsils were dual stained with EBER and either CD4 or CD8 to determine the number of T cells conjugated to EBER-positive cells,

Table 2 EBER-positive tonsil cell immunophenotype and T/NK cell conjugates

\begin{tabular}{lrrrc}
\hline & Tonsil 1 & Tonsil 2 & Tonsil 3 & Total (\%) \\
\hline EBER+ CD4+ $^{\mathrm{a}}$ & 16 & 9 & 8 & $33(4)$ \\
EBER+ CD4- & 51 & 421 & 367 & $839(96)$ \\
CD4+ conjugates & 35 & 229 & 202 & $466(53)$ \\
EBER+ CD8+ & 4 & 2 & 3 & $9(0.7)$ \\
EBER+ CD8- & 23 & 47 & 104 & $174(99.3)$ \\
CD8+ conjugates & 111 & 34 & 260 & $405(30.4)$ \\
EBER+ PEN5+ & 6 & 3 & 2 & $11(1.7)$ \\
PEN5+ conjugates & 30 & 14 & 18 & $62(9.8)$ \\
EBER+ CD138+ & 0 & 0 & 0 & 0 \\
\hline
\end{tabular}

${ }^{\mathrm{a}}$ The bright CD4 intensity expressed by these cells is inconsistent with a monocyte lineage.

Table 3 Comparative EBV latent protein expression in 20 randomly selected tonsils

\begin{tabular}{lrrr}
\hline Tonsil \# & \#EBNA1+ & \#EBNA2+ & \#LMP1+ \\
\hline 1 & 21 & 0 & 21 \\
2 & 15 & 17 & 15 \\
3 & 15 & 0 & 30 \\
4 & 2 & 2 & 4 \\
5 & 10 & 0 & 34 \\
6 & 6 & 10 & 22 \\
7 & 48 & 0 & ND \\
8 & 7 & 7 & 4 \\
9 & 21 & 0 & $\mathrm{ND}$ \\
10 & 90 & 0 & ND \\
11 & 12 & 28 & 41 \\
12 & 12 & 0 & 14 \\
13 & 10 & 7 & 13 \\
14 & 3 & 0 & 0 \\
15 & 13 & 0 & 112 \\
16 & 0 & 50 & 73 \\
17 & 45 & 44 & 18 \\
18 & 55 & 50 & 27 \\
19 & 50 & 0 & \\
20 & 21 & 11 & \\
& & & \\
Average & 23 & & \\
\hline
\end{tabular}

ND, not determined. as an in situ measure of T-cell-mediated immunity to EBV-positive cells in tonsils (Table 4). Although many EBER-positive cells (especially those with plasmacytoid features located within the crypt epithelium) were not in contact with T cells, most $(54-70 \%)$ were in direct contact with CD4-positive T cells, while only $30-32 \%$ of the EBER-positive cells were in direct contact with CD8-positive $\mathrm{T}$ cells. These results suggest that most of the EBERpositive cells in tonsils from patients without overt infectious mononucleosis are not under active cytotoxic T-cell attack. Furthermore, dual staining of sections with EBER and the TH2 marker CCR3 revealed large numbers of CCR3-positive TH2 cells in close contact with EBER-positive cells (data not shown). This result suggests that many of the EBVinfected B cells in tonsils from patients without IM are not only not targets of T-cell-mediated cytotoxicity, but are under the progrowth influence of Th2 cells.

\section{Discussion}

EBV infection is very common, with adult seroprevalence rates in excess of $90 \%$ worldwide. Following primary infection in the oropharynx, EBV persists in numerous anatomical sites including pharyngeal tonsils, adenoids, lymph nodes, and peripheral blood. In peripheral blood, virus is present in small resting memory B cells with latent gene expression limited to EBER, LMP2a, and perhaps EBNA1. ${ }^{7}$ In RT-PCR studies of isolated B cells from persistently infected tonsils, latent virus was found in all B-cell subsets, most commonly in IgD-memory $\mathrm{B}$ cells, but including $\mathrm{IgD}+$ naïve $\mathrm{B}$ cells and CD10 + germinal center B cells. ${ }^{8}$ In an RTPCR study, expression of latent genes EBNA1 and LMP was detected in all tonsil B-cell subsets, while growth-promoting EBNA2 expression was limited to the naïve $\operatorname{IgD}+\mathrm{B}$-cell subset. ${ }^{6}$ In contrast, in a study of acute IM tonsils, most EBV-positive cells (6080\%) expressed EBNA2, 20-30\% expressed LMP1 but not EBNA2, and $<10 \%$ expressed neither LMP1 nor EBNA2. ${ }^{9}$

Most of the EBV-infected cells (82\%) detected in this study of human tonsils appear to be small CD20-

Table 4 Relationship between localization, phenotype ${ }^{a}$, and T-cell conjugation of EBER+ tonsil cells

\begin{tabular}{|c|c|c|c|c|c|c|c|c|c|c|}
\hline & \multicolumn{4}{|c|}{ Intraepithelial } & \multicolumn{3}{|c|}{ Interfollicular } & \multicolumn{3}{|c|}{ Follicular } \\
\hline & $\begin{array}{c}\text { B cell } \\
(\%)\end{array}$ & $\begin{array}{c}\text { Tcell } \\
(\%)\end{array}$ & $\begin{array}{c}\text { Epithelial } \\
\text { cell (\%) }\end{array}$ & $\begin{array}{l}\text { Null cell } \\
(\%)\end{array}$ & $\begin{array}{c}\text { B cell } \\
(\%)\end{array}$ & $\begin{array}{c}\text { Tcell } \\
(\%)\end{array}$ & $\begin{array}{c}\text { Null } \\
\text { cell (\%) }\end{array}$ & $\begin{array}{c}\text { B cell } \\
(\%)\end{array}$ & $\begin{array}{c}\text { T cell } \\
(\%)\end{array}$ & $\begin{array}{l}\text { Null cell } \\
(\%)\end{array}$ \\
\hline T-cell conjugated & 10 & 0 & 0.7 & 0 & 41 & 0 & 1.3 & 6 & 0 & 0 \\
\hline T-cell unconjugated & 14 & 0.3 & 0.5 & 1.3 & 3.2 & 6.4 & 7.1 & 8 & 0 & 1.2 \\
\hline
\end{tabular}

${ }^{\mathrm{a}} \mathrm{B}$ cells are defined as CD20 positive, T cells as CD3 positive, epithelial cells as cytokeratin positive, and null cells as negative for all three markers. 
positive B cells, some with plasmacytoid features, located primarily in interfollicular regions and intimately associated with crypt epithelium. A little more than half $(59 \%)$ of the EBER-positive cells were located within the T-cell-rich interfollicular regions of the tonsils, while a significant number $(27 \%)$ were located within the lymphocyte-rich crypt epithelium. These results are consistent with results of a previous study of acutely infected tonsils from patients with IM in which EBER + B cells were mainly found in interfollicular areas often adjacent to crypt epithelium. ${ }^{9}$

In the present study, $15 \%$ of the EBER-positive cells, nearly all of which (92\%) stained as weakly $\mathrm{CD} 20+\mathrm{B}$ cells, were located within the B-cell-rich germinal centers of secondary follicles. Few follicles contained numerous EBER-positive cells. These results are consistent with previous reports of EBER-positive germinal center B cells in tonsils. ${ }^{10,11}$ It has been suggested that germinal center centroblasts may be a site of EBV persistence. ${ }^{12,13}$ The presence of EBV-positive B cells in germinal centers is reportedly more common in tonsils from areas endemic for EBV + lymphomas, leading to the suggestion that EBV-infected germinal center B cells may be more prone to malignant transformation, perhaps due to somatic hypermutation. ${ }^{11}$ In contrast to the presence of a few EBV-infected B cells in follicular germinal centers, no EBER-positive cells were detected within follicular mantle zones in the present study. Since mantle zone B cells typically express $\operatorname{IgD}$, this result is consistent with previous results in which most EBV-positive B cells in tonsils were shown to be IgD negative. ${ }^{8}$

Although the vast majority of the EBV-positive B cells in persistently infected tonsil are latent infected, virus replication presumably from productively infected BZLF1/VCA positive cells, many with plasmacytoid features, has been well documented. ${ }^{4,14-16}$ In the present study, a significant number $(11 \%)$ of the EBER-positive tonsil cells had plasmacytoid features and expressed dim to undetectable levels of B-cell antigen CD20, while many expressed EBV BZLF-1 protein characteristic of early lytic activation. These results are in agreement with those in a previous study in which many EBER-positive cells in IM tonsils were CD20negative, with plasmacytoid features, expressed the EBV immediate-early protein BZLF1, and were positive for the plasma cell antigen detected by monoclonal antibody VS38c. ${ }^{14}$ In the present study, expression of the plasma cell antigen CD138 was not detected in the EBER-positive plasmacytoid cells. Reduced expression of CD20 by EBV-infected plasmacytoid B cells is not surprising since both EBV infection and plasmacytoid differentiation are associated with reduced expression of CD20 on B cells. In a study of 37 EBV-transformed B lymphoblastoid cell lines, $11(30 \%)$ of the cell lines were negative for pan-B-cell markers CD19 and CD20. ${ }^{17}$ Also, it has been reported that up to $80 \%$ of the EBER-positive cells in the peripheral blood exhibit features of plasmacytoid differentiation. ${ }^{18}$ In the present study, numerous BZLF1-positive cells were detected within the tonsils, primarily within the lymphocyterich crypt epithelium. This result is consistent with previous reports documenting EBV replication in IM tonsils., ${ }^{4,15,16}$ Most of these cells appeared to be small- to medium-sized lymphocytes with plasmacytoid features. Given their location just beneath the crypt surface, it seems likely that these cells are the source of most of the infectious virus in saliva. The present results suggest that these small CD20-negative EBER-positive cells are more likely to be plasmacytoid B cells rather than fully mature plasma cells, and that these cells are responsible for nearly all of the EBV production in the tonsil.

In contrast to the large number of CCR3-positive $\mathrm{TH} 2$ cells in close contact with EBER-positive cells, relatively few CD8-positive T-cell - EBER + cell conjugates were identified. This intriguing result suggests that a majority of the EBV-infected cells in tonsils are not targets of T-cell-mediated antiviral cytotoxicity, but instead are surrounded by noncytotoxic Th2 cells that provide a microenvironment conducive to B-cell growth.

EBV infection of epithelial cells occurs in certain pathologic conditions, namely nasopharyngeal carcinoma and oral hairy leukoplakia. ${ }^{19-22}$ Zhang et $a l^{22}$ analyzed the presence of EBV DNA in nasopharyngeal carcinoma and nasopharyngitis and demonstrated that infection of non-neoplastic nasopharyngeal epithelial cells by EBV may occur in some patients with nasopharyngitis. Furakawa et $a l^{23}$ in 1990 exposed epithelial cells derived from primary cultures of adenoidal tissue to EBV and was able to detect EBV antigens in these epithelial cells. Also, epithelial cell lines susceptible to EBV infection in vitro have been described. ${ }^{24}$ Despite these findings, evidence for the presence of EBV within normal epithelial cells in vivo is conflicting. Some studies have reported the presence of EBV DNA in desquamated squamous epithelial cells and in tonsil epithelium. ${ }^{25-27}$ In contrast, other studies have reported no virus in desquamated epithelial cells or unequivocal evidence of EBV-infected tonsillar epithelial cells by EBER ISH and/or LMP1 immunostaining. ${ }^{4,14,28}$ The present results, based upon exhaustive histopathologic evaluation of many tonsil sections, provide direct in situ evidence that normal epithelial cells in tonsillar crypts from immunocompetent hosts may rarely be infected by EBV.

Both CD4- and CD8-positive T cells are susceptible to EBV infection in vitro, and there are many examples of EBV-infected T cells in certain pathologic conditions, including NK/T cell lymphoma, hemophagocytic lymphohistiocytosis, and chronic active EBV infection. ${ }^{29-34}$ The complement receptor CR2 (CD21) that serves as the EBV receptor on B cells is expressed by some peripheral blood T cells. ${ }^{35}$ 
However, results of EBER ISH combined with immunohistochemistry with T-cell-specific antibodies on IM tonsils have yielded conflicting results. ${ }^{4,14}$ In the present study, a few EBER-positive CD3-positive tonsillar T cells were identified. Nearly all of the EBER-positive $\mathrm{T}$ cells were small cells located within the interfollicular regions. Results of dual EBER/CD4 and EBER/CD8 immunostaining indicate that most of the infected T cells are CD4positive helper/inducer $\mathrm{T}$ cells rather than CD8positive cytotoxic T cells (Table 2). Although human monocyte cell lines are susceptible to EBV infection in vitro, ${ }^{36}$ EBER positivity could not be demonstrated on dim CD4-positive monocytes in this study.

NK cells are susceptible to EBV infection in vitro. ${ }^{37}$ Although NK cells, unlike T cells, do not normally express the CD21 EBV receptor, it has been demonstrated in vitro that NK cells bound to EBVinfected B cells may acquire the receptor via a so-called immunological synapse. ${ }^{38}$ Utilizing the NK-cell-specific monoclonal antibody PEN5 ${ }^{39}$ with EBER ISH, Trempat et $a l^{40}$ detected a few EBV-positive NK cells in reactive tonsils. In the present study utilizing the anti-PEN5 antibody, we also detected rare EBER-positive NK cells in these tonsils. These rare dual EBER/PEN5-positive cells were small and contracted with apoptotic features. In contrast, EBER-negative PEN5-positive NK cells were noted to be medium-sized lymphocytes with prominent dendritic cytoplasmic projections located just beneath the crypt epithelium (Figure 1i).

EBV-positive smooth muscle cell tumors have been described in immunocompromised pediatric transplant recipients. ${ }^{41-43}$ Although no EBV-positive endothelial cells or salivary gland cells were detected in the present study, rare BZLF-1-positive mesenchymal spindle cells of undetermined phenotype were identified (Figure 1r).

The rarity of EBV-infected non-B-cell types in tonsil suggests that under normal circumstances EBV infection of $\mathrm{T}$ cells, epithelial cells, and mesenchymal cells is not likely to be biologically relevant. The close physical association of EBERpositive B cells, some of which are lytically active (BZLF1 positive), with EBER-negative crypt epithelial cells and $T$ cells also suggests that transmission of EBV infection from B cells to non-B cells is a rare event. However, these results clearly demonstrate that the virus may rarely be found in non-B-cell types in reactive human tonsils, including $\mathrm{T}$ cells, NK cells, epithelial cells, and mesenchymal cells. These results provide a basis for further understanding of how, in some clinical settings characterized by inadequate immune surveillance, rare and unusual EBV-infected non-B-cells may, presumably following genetic mutation, give rise to EBV-positive NK/T cell lymphoma, hemophagocytic syndrome, nasopharyngeal carcinoma, and smooth muscle cell tumors.

\section{Acknowledgements}

We thank Barbara Hightower and Kerry Graves of the UTMB Immunopathology Laboratory and Linda Muelberger of the Sealy Center for Environmental Health and Medicine for their expert histopathologic assistance.

\section{References}

1 Henle G, Henle W, Clifford P, et al. Antibodies to EB virus in Burkitt's lymphoma and control groups. J Natl Cancer Inst 1969;43:1147-1157.

2 Niederman JC, Miller G, Pearson HA, et al. Infectious mononucleosis. Epstein-Barr-virus shedding in saliva and the oropharynx. N Engl J Med 1976;294:13551359.

3 Farrell PJ. Tumour viruses-could they be an Achilles' heel of cancer? Eur J Cancer 2002;38:1815-1816.

4 Anagnostopoulos I, Hummel $\mathrm{M}$, Kreschel $\mathrm{C}$, et al. Morphology, immunophenotype, and distribution of latently and/or productively Epstein-Barr virus-infected cells in acute infectious mononucleosis: implications for the interindividual infection route of Epstein-Barr virus. Blood 1995;85:744-750.

5 Faulkner GC, Krajewski AS, Crawford DH. The ins and outs of EBV infection. Trends Microbiol 2000; 8:185-189.

6 Babcock GJ, Thorley-Lawson DA. Tonsillar memory B cells, latently infected with Epstein-Barr virus, express the restricted pattern of latent genes previously found only in Epstein-Barr virus-associated tumors. Proc Natl Acad Sci USA 2000;97:12250-12255.

7 Miyashita EM, Yang B, Babcock GJ, et al. Identification of the site of Epstein-Barr virus persistence in vivo as a resting B cell. J Virol 1997;71:4882-4891.

8 Laichalk LL, Hochberg D, Babcock GJ, et al. The dispersal of mucosal memory B cells: Evidence from persistent EBV infection. Immunity 2002;16:745-754.

9 Kurth J, Spieker T, Wustrow J, et al. EBV-infected B cells in infectious mononucleosis: viral strategies for spreading in the B cell compartment and establishing latency. Immunity 2000;13:485-495.

10 Niedobitek G, Herbst H, Young LS, et al. Patterns of Epstein-Barr virus infection in non-neoplastic lymphoid tissue. Blood 1992;79:2520-2526.

11 Araujo I, Foss HD, Hummel M, et al. Frequent expansion of Epstein-Barr virus (EBV) infected cells in germinal centres of tonsils from an area with a high incidence of EBV-associated lymphoma. J Pathol 1999; 187:326-330.

12 Gregory CD, Kirchgens C, Edwards CF, et al. EpsteinBarr virus-transformed human precursor B cell lines: altered growth phenotype of lines with germ-line or rearranged but nonexpressed heavy chain genes. Eur J Immunol 1987;17:1199-1207.

13 Cirone M, De Maria R, D’Alessandro A, et al. EpsteinBarr virus DNA is present both in CD10/CD77 positive and negative subsets of human tonsillar lymphocytes. Cancer Lett 1995;89:125-128.

14 Niedobitek G, Agathanggelou A, Herbst $\mathrm{H}$, et al. Epstein-Barr virus (EBV) infection in infectious mononucleosis: virus latency, replication and phenotype of EBV-infected cells. J Pathol 1997;182:151-159. 
15 Babcock GJ, Decker LL, Volk M, et al. EBV persistence in memory B cells in vivo. Immunity 1998;9:395-404.

16 Ikeda T, Kobayashi R, Horiuchi M, et al. Detection of lymphocytes productively infected with Epstein-Barr virus in non-neoplastic tonsils. J Gen Virol 2000;81: 1211-1216.

17 Wrobleski JM, Copple A, Batson LP, et al. Cell surface phenotyping and cytokine production of EBV-transformed lymphoblastoid cell lines. J Immunol Methods 2002;264:19-28.

18 Robinson JB, Smith D, Miederman J. Plasmacytic differentiation of circulating Epstein-Barr virus-infected B lymphocytes during acute infectious mononucleosis. J Exp Med 1981;153:235-244.

19 Brooks L, Yao QY, Rickinson AB, et al. Epstein-Barr virus latent gene transcription in nasopharyngeal carcinoma cells: coexpression of EBNA1, LMP1, and LMP2 transcripts. J Virol 1992;66:2689-2697.

20 Greenspan JS, Greenspan D, Lennette ET, et al. Replication of Epstein-Barr virus within the epithelial cells of oral hairy leukoplakia, an AIDS-associated lesion. N Engl J Med 1985;313:1564-1571.

21 Niedobitek G, Young LS, Lau R, et al. Epstein-Barr virus infection in oral hairy leukoplakia: virus replication in the absence of a detectable latent phase. J Gen Virol 1991;72:3035-3046.

22 Zhang HJ, Qu G, Deng ZW, et al. Epstein-Barr virus DNA in nasopharyngeal biopsies. Virus Res 1989;12: 53-59.

23 Furukawa M, Sakashita H, Kato C, et al. Epstein-Barr virus infection of epithelial cells derived from primary cultures of adenoidal tissue. Eur Arch Otorhinolaryngol 1990;247:109-113.

24 Li QX, Young LS, Niedobitek G, et al. Epstein-Barr virus infection and replication in a human epithelial cell system. Nature 1992;356:347-350.

25 Lemon SM, Hutt LM, Shaw JE, et al. Replication of EBV in epithelial cells during infectious mononucleosis. Nature 1977;70:268-270.

26 Sixbey JW, Nedrud JG, Raab-Traub N, et al. EpsteinBarr virus replication in oropharyngeal epithelial cells. N Engl J Med 1984;310:1225-1230.

27 Kobayashi R, Takeuchi H, Sasaki M, et al. Detection of Epstein-Barr virus infection in the epithelial cells and lymphocytes of non-neoplastic tonsils by in situ hybridization and in situ PCR. Arch Virol 1998;143: 803-813.

28 Niedobitek G, Agathanggelou A, Steven N, et al. Epstein-Barr virus (EBV) in infectious mononucleosis: detection of the virus in tonsillar B lymphocytes but not in desquamated oropharyngeal epithelial cells. Mol Pathol 2000;53:37-42.

29 Guan M, Zhang RD, Wu B, et al. Infection of primary CD4+ and CD8+ T lymphocytes by Epstein-Barr virus enhances human immunodeficiency virus expression. J Virol 1996;70:7341-7346.

30 Quintanilla-Martinez L, Kumar S, Fend F, et al. Fulminant EBV+ T-cell lymphoproliferative disorder following acute/chronic EBV infection: a distinct clinicopathologic syndrome. Blood 2000;96:443-451.

31 Dreyfus DH, Nagasawa M, Pratt JC, et al. Inactivation of NF-kappaB by EBV BZLF-1-encoded ZEBRA protein in human T cells. J Immunol 1999;163:6261-6268.

32 Kanavaros P, Lescs MC, Briere J, et al. Nasal T cell lymphoma: a clinicopathologic entity associated with peculiar phenotype and with Epstein-Barr virus. Blood 1993;81:2688-2695.

33 Kawaguchi H, Miyashita T, Herbet H, et al. EpsteinBarr virus-infected $\mathrm{T}$ lymphocytes in Epstein-Barr virus-associated hemophagocytic syndrome. J Clin Invest 1993;92:1444-1450.

34 Kikuta H, Taguchi Y, Tomizawa K, et al. Epstein-Barr virus genome-positive $\mathrm{T}$ lymphocytes in a boy with chronic active EBV infection associated with Kawasaki-like disease. Nature 1988;333:455-457.

35 Fisher E, Delibrias C, Kazatchkine MD. Expression of the CR2 (the C3dg/EBV receptor, CD21) on normal human peripheral blood $\mathrm{T}$ lymphocytes. J Immunol 1991;146:865-872.

36 Masy E, Adriaenssens E, Montpellier C, et al. Human monocytic cell lines transformed in vitro by EpsteinBarr virus display a type II latency and LMP-1dependent proliferation. J Virol 2002;76:6460-6472.

37 Isobe Y, Sugimoto K, Yang L, et al. Epstein-Barr virus infection of human natural killer cell lines and peripheral blood natural killer cells. Cancer Res 2004;64:2167-2174.

38 Tabiasco J, Vercellone A, Meggetto F, et al. Acquisition of viral receptor by NK cells through immunological synapse. J Immunol 2003;170:5993-5998.

39 Vivier E, Munroe M, Ariniello P. Identification of tissue-infiltrating lymphocytes expressing PEN5, a mucin-like glycoprotein selectively expressed on natural killer cells. Am J Pathol 1995;146:409-418.

40 Trempat P, Tabiasco J, Andre P, et al. Evidence for early infection of nonneoplastic natural killer cells by Epstein-Barr virus. J Virol. 2002;76:11139-11142.

41 Prevot S, Neris J, de Saint Maur PP. Detection of Epstein Barr virus in an hepatic leiomyomatous neoplasm in an adult human immunodeficiency virus 1-infected patient. Virchows Arch 1994;425:321-325.

42 Lee ES, Locker J, Nalesnik M, et al. The association of Epstein-Barr virus with smooth-muscle tumors occurring after organ transplantation. N Engl J Med 1995; 332:19-25.

43 McClain KL, Leach CT, Jenson HB, et al. Association of Epstein-Barr virus with leiomyosarcomas in children with AIDS. N Engl J Med 1995;332:12-18. 